\title{
Analysis on Immune Tolerance and Resistance Mechanism of Cryptococcus albidus of AIDS Patients with Opportunistic Cryptococci Infection
}

\author{
Xiaobing Yang1*, Guosheng Su${ }^{2 * \#, ~ L i d a ~} \mathrm{Mo}^{2}$ \\ ${ }^{1}$ The Guangxi Zhuang Autonomous Region, Longtan Hospital of the People's Republic of China, Liuzhou, China \\ ${ }^{2}$ The Fourth People's Hospital Clinical Laboratory Nanning Guangxi, Guangxi Medical University Affiliated to \\ Infectious Diseases Hospital of Nanning, Guangxi AIDS Clinical Treatment Center, Nanning, China \\ Email: " suguoshengv@sina.com
}

Received 8 August 2015; accepted 26 September 2015; published 29 September 2015

Copyright (C) 2015 by authors and Scientific Research Publishing Inc.

This work is licensed under the Creative Commons Attribution International License (CC BY). http://creativecommons.org/licenses/by/4.0/

(c) (i) Open Access

\begin{abstract}
Objective: To explore more about the immune tolerance and drug resistance of white Cryptococci albidus in AIDS patients with opportunistic Cryptococcus infection. Methods: To analyze drug resistance of the samples of white Cryptococcus albidus extracted from opportunistic infection AIDS patients in the certain infection area from October 2011 to December 2014. Results: After analyzing two samples of Cryptococcus albidus from 885 cases with opportunistic infection, we found that one of the samples do resist to ten common antibiotics. They were fluconazole, flu-cytosine, fluconazole, caspofungin, amphotericin B, MI miconazole, terbinafine, ketoconazole and itraconazole. The other one was sensitive to voriconazole, but resistant to the rest of the drug. Two strains of bacteria were inoculated into the animals in vivo and their DNA was extracted to carry out the genotyping analysis. The results showed that different degrees of resistance gene amplification bands were found in the 10 kinds of antibiotics. Conclusion: Although there were few opportunistic infection Cryptococcus albidus in AIDS patients, it was easy to show its resistance to drugs. Therefore, great attention should be paid to it for the medical workers.
\end{abstract}

\section{Keywords}

AIDS Patients, Opportunistic Infections, Cryptococcus albidus, Analysis of Drug Resistance

\footnotetext{
"The first authors.

"Corresponding author.
}

How to cite this paper: Yang, X.B., Su, G.S. and Mo, L.D. (2015) Analysis on Immune Tolerance and Resistance Mechanism of Cryptococcus albidus of AIDS Patients with Opportunistic Cryptococci Infection. Advances in Infectious Diseases, 5, 118-124. http://dx.doi.org/10.4236/aid.2015.53014 


\section{Introduction}

It occurs occasionally to AIDS patients that they get opportunistic infection of pathogenic bacteria, especially tuberculosis infection of Candida albidus, Mycobacterium co-infected with tuberculosis, Escherichia coli, G Klebsiella, Pseudomonas aeruginosa bacteria and so on [1]-[4]. In the Cryptococcus, Cryptococcus neoformans is the main, but Cryptococcus albicans is rare. At present, there has been no related report on AIDS co-infected with Cryptococcus albicans at home and abroad. After screening large-scale samples from the 885 cases of AIDS opportunistic infection patients, two cases of patients with Cryptococcus albidus are extracted. After analyzing these two samples, we find that one of the samples do resist to ten common antibiotics. They are fluconazole, flu-cytosine, fluconazole, caspofungin, amphotericin B, MI miconazole, terbinafine, ketoconazole and itraconazole. And the other one was sensitive to voriconazole, but resistant to the rest of the drug. Two strains of bacteria are inoculated into the animals in vivo and their DNA is extracted to carry out the genotyping analysis. The results show that different degrees of resistance gene amplification bands are found in the 10 kinds of antibiotics. The results of the study are as follows.

\section{Materials and Methods}

\subsection{Data Sources}

Those 885 cases of opportunistic infections in AIDS patients were selected from Guangxi AIDS clinical treatment center in Nanning from October 2011 to December 2014, including 658 cases of males, 227 cases of women, and they are at the age of 21 to 76. Their average age was 42.5, 3.5 years more or less. All the specimens have been approved by medical ethics commission of AIDS clinical treatment center in Nanning, Guangxi and got consent of the patients themselves for laboratory study.

\subsection{Research Methods}

First of all, collect patients' blood, body fluids, secretions, feces, tissue fluid and other specimens, and then send them to the center of medical experiment. All specimens were selected for selective inoculations in common blood culture flask, common blood culture dish, Makanke culture dish, SS culture dish, culture medium or KIA and so on. And then other related smear microscopy was carried out. After that, extract some growing bacteria for purifications of the vaccination bacteria. Later, identification and drug susceptibility tests were performed. Select two cases with Cryptococcus albidus cultures and inoculate them in the brain cavities of each experimental rabbits for 15 days. And then observe the changes of the rabbits' immune system and analyze the immunologic mechanism of Cryptococcus neoformans to the hosts. Next, extract the rabbits' cerebrospinal fluid (CSF) for DNA detection and analysis of antimicrobial resistance genes. Finally, treat the experimental animals with voriconazole and observe the effect of treatment.

\subsection{Reagents, Instruments and Experimental Animals}

The reagents and instruments used in this study have passed the quality checks and are approved by the authority of the Department of Clinical Research. The experimental animal rabbits are from the United States. Cyclophosphamide instrument are mainly BacT ALTERT 3D120 from BioMerieux. The automatic blood culture instruments are provided by the French BioMerieux Company, and the TIANDIREN TDR-1002 bacteria identification of susceptibility analyzer was provided by Hunan Changsha TIANDIREN Biotechnology Co., Ltd., The carbon dioxide incubator, bacterial culture box, fungal culture curing box and mycobacterial culture box were provided by the Chinese famous brand companies of medical apparatus and instruments. All of Hemoline and the agar plates were provided by Zhengzhou Antu bioengineering Co., Ltd., including the blood agar plate, Jimmy Kang Kai agar, chocolate blood agar, SS agar and CANDIDAID. Neutral Roche culture tube and Mycobacterium drug susceptibility Roche culture tube were provided by Zhuhai beisuo Biological Technology Co. Ltd. Salmonella serotypes and shigella serotypes were provided by Ningbo Tianrun Biological Pharmaceutical Co., Ltd., Kligler iron agar, nutrient broth agar, and AupuToshin paper, bacitracin were supplied by Hangzhou Days and microbial Reagent Co., Ltd. All the reagent kits were provided by Changsha TIANDIREN biotechnology limited company including Enterobacteriaceae kit, non-fermentative bacteria, Staphylococcus aureus kit, Streptococcus kit, Neisseria or Haemophilus kit and yeast like fungus detection kit. All the reagents were used 
within the period of validity. A pair of primers P4501 and P4502 was designed. The primer sequence of P4501 was 5'-ATG ACT GATCAA GAA ATYGCTAA-3' and P4502 was 5'-TAA CCTGGAGAAACY AAAAC-3'. The primers were provided by Shanghai engineering company. The small molecular Marker was supported by the United States MBI company. The extract of DNA was supplied by the American OBIGGENE company, and the PCR was American ABI7500.

\subsection{Quality Control}

Strains of Candida albicans (ATCC90028), Candida albicans (ATCC6258), Staphylococcus aureus (ATCC25923), Escherichia coli (ATCC25922), Pseudomonas aeruginosa (ATCC27853) and fecal intestinal bulb (ATCC29212) were provided by the Guangxi clinical test center, while the standard attenuated strain of Mycobacterium tuberculosis (ATCC25177) was provided by Guangxi CDC. The results were strictly acquired through the latest standards of the American clinical laboratory standardization association (CLSI).

\subsection{Statistical Data Processing}

Statistical software SPSS17.0 was used for statistical analysis, the difference was statistically significant with $P$ $<0.05$.

\section{Results}

\subsection{The Distribution of AIDS Opportunistic Infection of Pathogenic Bacteria}

In the extraction of 885 cases of AIDS opportunistic infection of the pathogenic bacteria strains, cultivation of fungi was 356 cases, accounting for 40.23\%, 141 cases with gram positive cocci, accounting for $15.93 \%$, Enterobacteriaceae was 276 cases, accounting for 31.19\%, non-fermentation bacteria 95 cases, accounted for 10.73\%, and gram positive bacilli 17 cases, accounted for $1.92 \%$. Besides, there were 98 cases of Mycobacterium tuberculosis co-infection, which were extracted from the opportunistic infection examples, accounting for $11.07 \%$. And there were two cases of Cryptococcus albidus extracted from fungi bacteria, accounting for $0.226 \%$ of the total number of opportunistic infection pathogenic bacteria. After analyzing drug sensitivity of these two samples, we found that one of the samples (hereinafter referred to as NO. 1 strains) do resist to ten common antibiotics. They are fluconazole, flu-cytosine, fluconazole, caspofungin, amphotericin B, MI miconazole, terbinafine, ketoconazole and itraconazole. And the other one (hereinafter referred to as NO. 2 strains) was sensitive to voriconazole, but resistant to the rest of the drug. The distribution of AIDS opportunistic infection of pathogenic bacteria and resistance to 10 kinds of antibiotics are as follows (Table 1, Table 2).

\subsection{The Changes of Immune System in Inoculation of the Two Cryptococcus albidus with the Experimental Animals}

Select two cases with Cryptococcus albidus cultures and inoculate them in the brain cavities of each experimental rabbits for 15 days.

The levels of immune parameters in the two groups of immune animals were changed accordingly. The levels of IgG and IFN- $\gamma$ in NO. 1 strain groups were significantly lower than NO. 2 strain's, while the levers of IL-4 were higher than those of NO. 2 strain's. As $P$ is less than 0.05 , there was statistically significant. However, there was no statistically significant in the rest of indicators such as IgA, IgM, TNF- and IL-10 levels, because $P$ was more than 0.05 (Table 3).

\subsection{The Expression of Drug Resistance Genes of the Two Cases with Cryptococcus albidus}

Two strains of bacteria were inoculated into the animals in vivo and their DNA was extracted to carry out the genotyping analysis. The results showed that different degrees of resistance gene amplification bands were found in the 10 kinds of antibiotics. For more details please look at Figure 1 and Table 4 below.

\section{Discussions}

It is very common to see AIDS opportunistic infection pathogens which will have bad influence on the patient's 
Table 1. The distribution of 885 cases of AIDS opportunistic infection of pathogenic bacteria.

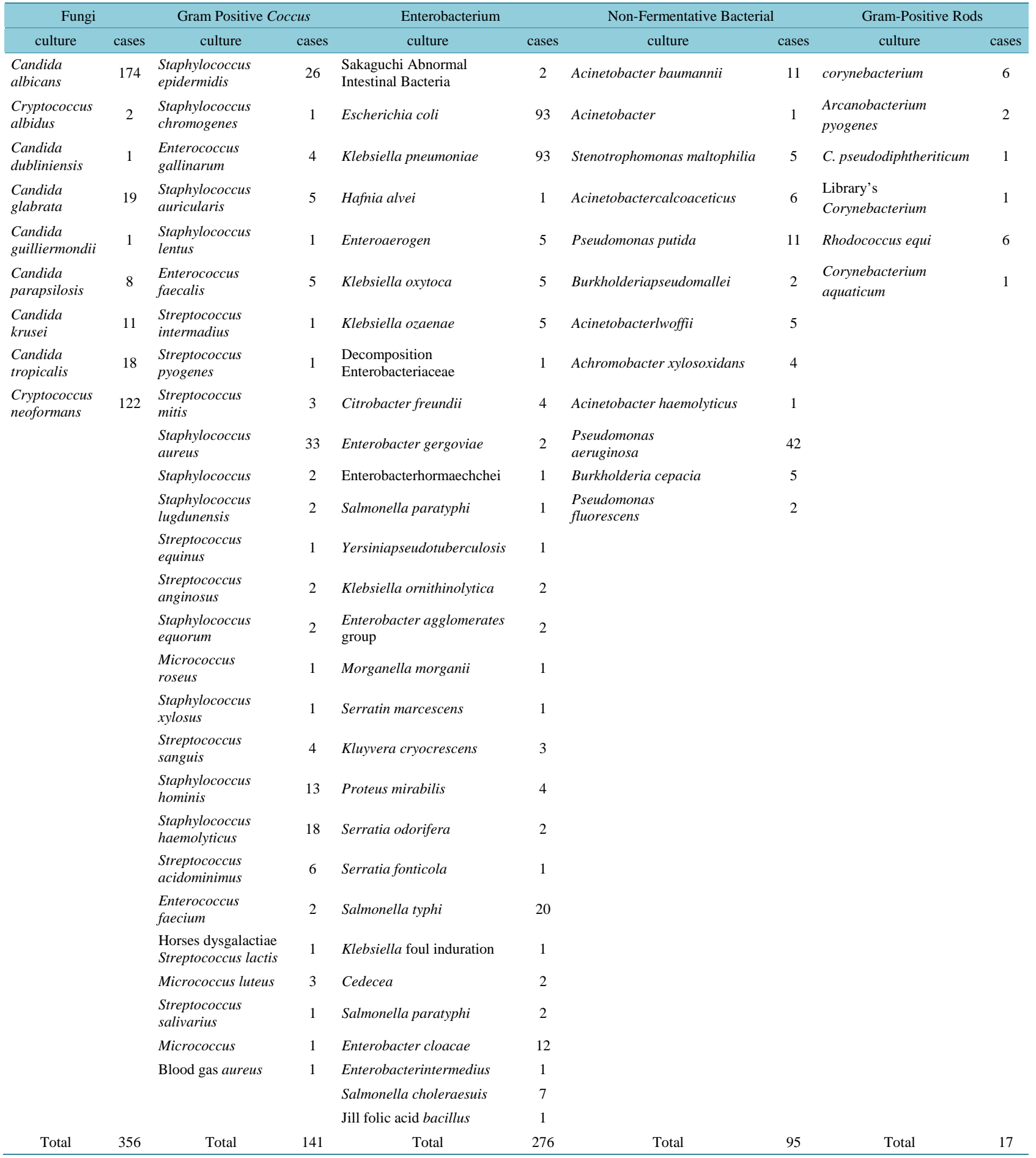

Table 2. The resistance of the two Cryptococcus albidus to 10 kinds of antibiotics (resistance for R, sensitive for S).

\begin{tabular}{ccccccccc}
\hline Bacteria names & voriconazole & flucytosine & fluconazole & caspofungin & amphotericin B & miconazole & terbinafine & ketoconazole \\
No. 1 strains & $\mathrm{R}$ & $\mathrm{R}$ & $\mathrm{R}$ & $\mathrm{R}$ & $\mathrm{R}$ & $\mathrm{R}$ & $\mathrm{R}$ & $\mathrm{R}$ \\
No. 2 strains & $\mathrm{S}$ & $\mathrm{R}$ & $\mathrm{R}$ & $\mathrm{R}$ & $\mathrm{R}$ & $\mathrm{R}$ & $\mathrm{R}$ & $\mathrm{R}$ \\
\hline
\end{tabular}


Table 3. The changes of serum immunoglobulin and immune related molecules in the two groups' patients $(\chi$ is more or less S).

\begin{tabular}{ccccc}
\hline items & No. 1 strains & No. 2 strains & $t$ & $P$ \\
\hline $\operatorname{IgA}(\mathrm{g} / \mathrm{L})$ & $1.56 \pm 0.76$ & $1.62 \pm 0.73$ & 0.1800 & 0.4296 \\
$\operatorname{IgG}(\mathrm{g} / \mathrm{L})$ & $6.23 \pm 0.72$ & $7.04 \pm 0.63$ & 2.6773 & 0.0077 \\
$\operatorname{IgM}(\mathrm{g} / \mathrm{L})$ & $1.36 \pm 0.58$ & $1.42 \pm 0.69$ & 0.2105 & 0.4178 \\
IFN- $\gamma(\mathrm{pg} / \mathrm{ml})$ & $8.26 \pm 1.32$ & $9.36 \pm 1.22$ & 1.9353 & 0.0344 \\
TNF- $\alpha(\mathrm{pg} / \mathrm{ml})$ & $2.13 \pm 0.56$ & $2.06 \pm 0.45$ & 0.3081 & 0.3808 \\
IL-4(pg/ml) & $4.05 \pm 0.36$ & $3.68 \pm 0.39$ & 2.2045 & 0.0204 \\
IL-10(pg/ml) & $2.35 \pm 0.59$ & $2.18 \pm 0.63$ & 0.6228 & 0.2706 \\
\hline
\end{tabular}

Table 4. The analysis results of Cryptococcus genotyping mutations resistance in the two strains (n, \%),

\begin{tabular}{ccccc}
\hline \multirow{2}{*}{ drug names } & \multicolumn{2}{c}{ No. 1 stains } & \multicolumn{2}{c}{ No. 2 strains } \\
\cline { 2 - 5 } & sensitivity(\%) & resistance(\%) & sensitivity(\%) & resistance(\%) \\
\hline voriconazole & $0.0(0 / 10)$ & $100.0(10 / 10)$ & $100.0(10 / 10)$ & $0(0 / 10)$ \\
flucytosine & $0.0(0 / 10)$ & $100.0(10 / 10)$ & $0(0 / 10)$ & $100.0(10 / 10)$ \\
fluconazole & $0.0(0 / 10)$ & $100.0(10 / 10)$ & $0(0 / 10)$ & $100.0(10 / 10)$ \\
caspofungin & $0.0(0 / 10)$ & $100.0(10 / 10)$ & $0(0 / 10)$ & $100.0(10 / 10)$ \\
amphotericin B & $0.0(0 / 10)$ & $100.0(10 / 10)$ & $0(0 / 10)$ & $100.0(10 / 10)$ \\
miconazole & $0.0(0 / 10)$ & $100.0(10 / 10)$ & $0(0 / 10)$ & $100.0(10 / 10)$ \\
terbinafine & $0.0(0 / 10)$ & $100.0(10 / 10)$ & $0(0 / 10)$ & $100.0(10 / 10)$ \\
ketoconazole & $0.0(0 / 10)$ & $100.0(10 / 10)$ & $0(0 / 10)$ & $100.0(10 / 10)$ \\
itraconazole & $0.0(0 / 10)$ & $100.0(10 / 10)$ & $0(0 / 10)$ & $100.0(10 / 10)$ \\
nystatin & $0.0(0 / 10)$ & $100.0(10 / 10)$ & $0(0 / 10)$ & $100.0(10 / 10)$
\end{tabular}

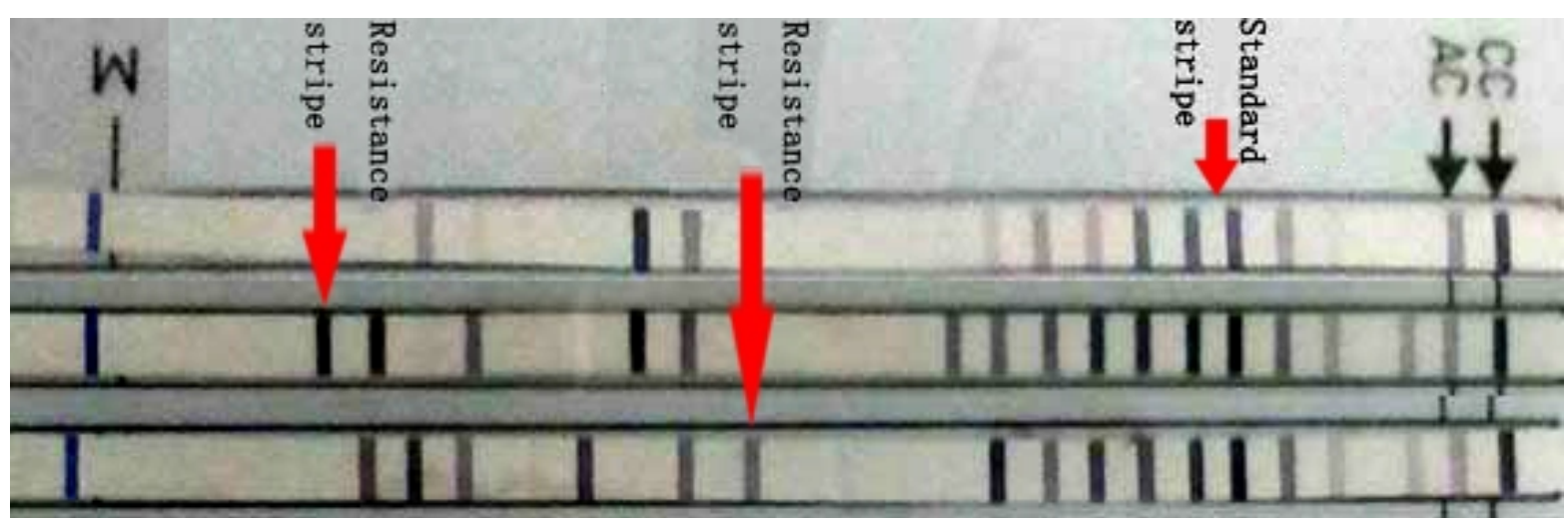

Figure 1. Standard strips and resistance bands.

life quality or even threaten the lives of patients if they are not treated in time [5]-[8]. Therefore, to understand the distribution and drug resistance analysis of opportunistic infection of pathogenic bacteria can be beneficial to the clinical treatment and prognosis. The research results showed that 84 species of bacteria were cultivated, which almost included all of the bacteria, bacteria genera and species in these 885 cases of opportunistic infections of pathogenic bacteria. Among these cultivated bacteria, fungal infected was particularly severe, whose in- 
fection rate reached $40.23 \%$, followed by the Enterobacteriaceae, reaching $31.19 \%$, while in patients with fungal infection, 2 cases with Cryptococcus albidus were extracted from them, reaching $0.226 \%$. According to these results, it is not hard to find that due to the low immune function of patients with AIDS, all kinds of pathogenic bacteria invade the body, which makes the treatment more complex. However, it is extremely rare to see Cryptococcus albidus in opportunistic infection of pathogenic bacteria. In this study, it is really difficult to detect 2 cases of Cryptococcus albidus from 885 patients. And thus, researchers should pay more attention to this subject. In the test of 10 commonly used antibiotics susceptibility, No. 1 bacteria strains were resistant to all drugs, while strains No. 2 were sensitive to voriconazole, but showed the resistance to the rest. Judging from these results, it can be found that Cryptococcus albidus resistance rate is extremely high in AIDS opportunistic infection and causes poor treatment effect.

The result indicates that the higher resistance of Cryptococcus albidus, the bigger effect of related immune indexes will occurs. When select two cases with Cryptococcus albidus cultures and inoculate them in the brain cavities of each experimental rabbits for 15 days the levels of immune parameters in the two groups of immune animals were changed accordingly, and in all immune indexes like IgA, IgM, IFN- $\gamma$, TNF- $\alpha$, IL-4, IL-10, the lever of IgG and IFN- $\gamma$ in NO. 1 strain groups were significantly lower than NO. 2 strain's, while the levers of IL-4 were higher than those of NO. 2 strains'. As $P$ is less than 0.05 , there was statistically significant. However, there was no statistically significant in the rest of indicators such as IgA, IgM, TNF- and IL-10 levels, because $P$ was more than 0.05 .

The results still showed that after analyzing the drug resistance of the cerebrospinal fluid (CSF), we found that the drug resistance of 2 strains of Staphylococcus was consistent with the results in routine culture sensitivity test. In No. 1 bacteria strains of ten cases of the experimental animals, there were 8 cases in the cerebrospinal fluid of the animals, and 2 cases with deletion type of wild drug resistance, while in strains No. 2 group, 10 cases of experimental animals CSF samples were shown to be sensitive to voriconazole, but resistance to the rest of the 9 kinds of antibiotic, among which there were 5 cases resulting in mutation resistance, 4 cases with loss of wild-type resistance. The strains No. 2 were resistant to the 9 kinds of antibiotics. What mentioned above indicates that the lever of resistance of Cryptococcus albidus in AIDS opportunistic infection is high and drug resistance of genetic-typing was consistent with the results in routine culture sensitivity test.

\section{Conclusion}

Although there is relatively rare of AIDS patients with opportunistic infection of Cryptococcus albidus, it has brought disastrous threat to patients, because white Cryptococcus neoformans are resistant to 10 kinds of commonly used antibiotics. The conventional culture resistance test results are consistent with the gene sub type resistance analysis results. Its resistance to drugs is rarely high, which brings trouble to clinical treatment. Therefore, medical workers should pay more attention to it.

\section{Limitations of Research}

Although this study has made some achievements, there are still some limitations, for example, in the process of the experiment, many interference factors are influenced much on the materials in the study. It is difficult to fully guarantee the quality of testing. In addition, laboratory conditions and work environment will also affect the test results. What we can do is to maximize the avoidance of interference.

\section{Acknowledgements}

This study got much help and support from many departments and individuals, such as the projects of science and technology, and the Guangxi AIDS clinical treatment center (Nanning), and other personnel not involved in this project research, all of them offered a great support and help in this research. Now here, all of members of this research group show our deepest appreciation to them, and wish them good health and everything goes well.

\section{Fund Project}

Nanning Guangxi scientific research and technology development plan (No. 20143154); the Guangxi Zhuang Autonomous Region health department self-financing research project (No. Z2014574). 


\section{References}

[1] Chen, M., Pan, W.H. and Boekhout, T. (2013) Cryptococcus gattii Infections in China: Extent of the Problem? Chinese Medical Journal, 126, 203-205.

[2] Hong, L.X., Song, W.J., Bo, F.X., et al. (2014) Cryptococcus neoformans and Gerth Clinical Molecular Epidemiology of Cryptococcus in Shenzhen. Chinese Journal of Infectious, 32, 204-208.

[3] Alanis, E., Macdougall, L., Kidd, S., et al. (2010) Epidemiology of Cryptococcus gattii, British Columbia, Canada, 1999-2007. Emerging Infectious Diseases, 16, 251-257. http://dx.doi.org/10.3201/eid1602.090900

[4] Kamoto, K., Hatakeyama, S., Itoyama, S., et al. (2010) Cryptococcus gattii Genotype VGIIa Infection in Man, Japan, 2007. Emerging Infectious Diseases, 16, 1155-1157. http://dx.doi.org/10.3201/eid1607.100106

[5] Li, L.X., Yuan, T., Zhen, S.Y., et al. (2012) The Construction of Expression Vector and Identification in MISlGene siRNA of Cryptococcus neoformans. Chinese Journal of Mycology, 7, 17-19.

[6] Fang, M.Y., Xian, F.Z., Wei, F., et al. (2015) Construction and Application of Cryptococcus Infection in Vitro BloodBrain Barrier Model. Chinese Journal of Mycology, 10, 92-95.

[7] Srinivasan, B., Kolli, A.R., Esch, M.B., et al. (2015) TEER Measurement Techniques for in Vitro Barrier Model Systems. Journal of Laboratory Automation, 20, 107-126. http://dx.doi.org/10.1177/2211068214561025

[8] Wuest, D.M., Wing, A.M. and Lee, K.H. (2013) Menbrane Configuration Optimization for a Murine in Vitro BloodBrain Barrier Model. Journal of Neuroscience Methods, 212, 211-221.

http://dx.doi.org/10.1016/j.jneumeth.2012.10.016 